\title{
Power Comparison of Independence Test for the Farlie-Gumbel-Morgenstern Family
}

\author{
Amini, M. ${ }^{1, a}$, Jabbari, $\mathrm{H}^{a}{ }^{\text {, Mohtashami Borzadaran, G.R. }}{ }^{a}$, Azadbakhsh, M. ${ }^{b}$ \\ ${ }^{a}$ Department of Statistics, Ordered and Spatial Data Center of Excellence, \\ Ferdowsi University of Mashhad; ${ }^{b}$ Department of Mathematics, University of Damghan
}

\begin{abstract}
Developing a test for independence of random variables $X$ and $Y$ against the alternative has an important role in statistical inference. Kochar and Gupta (1987) proposed a class of tests in view of Block and Basu (1974) model and compared the powers for sample sizes $n=8,12$. In this paper, we evaluate Kochar and Gupta (1987) class of tests for testing independence against quadrant dependence in absolutely continuous bivariate Farlie-GambelMorgenstern distribution, via a simulation study for sample sizes $n=6,8,10,12,16$ and 20. Furthermore, we compare the power of the tests with that proposed by Güven and Kotz (2008) based on the asymptotic distribution of the test statistics.
\end{abstract}

Keywords: Negative and positive quadrant dependence, Farlie-Gambel-Morgenstern distribution, U-Statistics.

\section{Introduction}

Dependence relations between random variables is one of the most widely studied topics in probability theory and statistics. Among them, our concentration is on positive and negative quadrant dependence (PQD and NQD), that are the most useful concept of dependence. The notations of these concepts are introduced by Lehmann (1966). Both PQD and NQD are qualitative forms of dependence and indicated whether or not a pair of random variables exhibits positive or negative dependence. Thus, for many purposes, in addition to the knowledge of the nature of dependence, it is also important to test independence against quadrant dependence. Let $(X, Y)$ be an absolutely continuous random vector with joint distribution function $F(x, y)$, survival function $\bar{F}(x, y)=P[X>x, Y>y]$, marginal distribution functions $F_{1}(x)$ and $F_{2}(y)$ and survival functions $\bar{F}_{1}(x)=P[X>x]$ and $\bar{F}_{2}(y)=P[Y>y]$. The random vector $(X, Y)$ is said quadrant dependent, if $F(x, y) \neq F_{1}(x) F_{2}(y)$ for all $x, y \in R$. In particular, the random vector $(X, Y)$ is said negative (positive) quadrant dependent, if $F(x, y) \leq(\geq$ ) $F_{1}(x) F_{2}(y)$ or equivalently $\bar{F}(x, y) \leq(\geq) \bar{F}_{1}(x) \bar{F}_{2}(y)$ for every $x, y \in R$. The dependence is strict if the inequalities hold for at least one pair of $(x, y)$.

Many statisticians are interested in finding the best test statistics for testing independence hypothesis $H_{0}: F(x, y)=F_{1}(x) F_{2}(y)$ against the alternative $H_{1}: F(x, y)<F_{1}(x) F_{2}(y)$ (or $H_{2}: F(x, y)>$ $\left.F_{1}(x) F_{2}(y)\right)$ for all real numbers $x, y$. There are many tests available in the literature to test independence against dependence, for example, Kendall's tau test and Spearman's rank correlation test (see Gibbons, 1971; Koroljuk and Borovskich, 1994; Serfling, 1980). Kochar and Gupta (1987, 1990) proposed some competitors of Kendall's tau coefficient for testing independence against the alternative of strictly positive quadrant dependence. Moreover, they studied simulation work to estimate the power

\footnotetext{
This research was supported by a grant from Ferdowsi University of Mashhad (No. MS87120AMI).

${ }^{1}$ Corresponding author: Associated Professor, Department of Statistics, Ordered and Spatial Data Center of Excellence, Ferdowsi University of Mashhad, Mashhad, Iran. E-mail: m-amini@um.ac.ir
} 
of the tests for small sample of sizes in absolutely continuous bivariate exponential distribution based on Block and Basu (1974) model. Shetty and Pandit (2003) considered a class of distribution-free tests for testing independence against PQD, which is a generalization of Kochar and Gupta (1990) results. Many papers such as Modarres (2007) and Hanagal and Kale (1991) are published in this area. Güven and Kotz (2008) introduced a test of independence against quadrant dependence for a pair of absolutely continuous random variables jointly distributed according to the generalized FarlieGambel-Morgenstern(FGM) distribution and derived an approximation of the test statistic distribution for large sample sizes.

In this paper, we apply in view of Kochar and Gupta (1987), the class of tests for testing independence against $H_{1}$ or $H_{2}$ in absolutely continuous bivariate FGM distributions. Also, we obtain the best test statistics for each samples of size $n=6,8,10,12,16$ and 20 via comparing our simulation results. Furthermore, we compute the power of the tests based on asymptotic distribution of the test statistics and also compare our results with those of Güven and Kotz (2008) for sample sizes $n=10,14,16,20$ and 50 in special cases.

\section{Preliminaries}

Let $k$ be a fixed integer and consider $d_{k}(x, y)=F^{k}(x, y)-F_{1}^{k}(x) F_{2}^{k}(y), \forall x, y \in R$ (according to notations of Kochar and Gupta (1987)). It is obvious that $d_{k}(x, y)<0(>0)$ if $H_{1}\left(H_{2}\right)$ is true and $d_{k}=0$ if $H_{0}$ is true, for all $k \geq 1$, that means significant of independence against NQD (PQD).

The following index is given to measure the deviation between $H_{0}$ and $H_{1}\left(H_{2}\right)$

$$
D_{k}=\int_{R^{2}} d_{k}(x, y) d F(x, y)=D_{1 k}-D_{2 k},
$$

where

$$
\begin{aligned}
P\left[\max _{1 \leq i \leq k} X_{i} \leq X_{k+1}, \max _{1 \leq i \leq k} Y_{i} \leq Y_{k+1}\right] & =\int_{R^{2}} P\left[\max _{1 \leq i \leq k} X_{i} \leq X_{k+1}, \max _{1 \leq i \leq k} Y_{i} \leq Y_{k+1} \mid X_{k+1}=x, Y_{k+1}=y\right] d F(x, y) \\
& =\int_{R^{2}} P\left[\max _{1 \leq i \leq k} X_{i} \leq x, \max _{1 \leq i \leq k} Y_{i} \leq y \mid X_{k+1}=x, Y_{k+1}=y\right] d F(x, y) \\
& =\int_{R^{2}} P\left[\bigcap_{i=1}^{k}\left\{X_{i} \leq x, Y_{i} \leq y\right\}\right] d F(x, y) \\
& =\int_{R^{2}} F^{k}(x, y) d F(x, y)=D_{1 k}
\end{aligned}
$$

and

$$
D_{2 k}=\int_{R^{2}} F_{1}^{k}(x) F_{2}^{k}(y) d F(x, y)=\int_{R^{2}} \bar{F}(x, y) d F_{1}^{k}(y) d F_{2}^{k}(y), \quad \forall k \geq 1 .
$$

The equality of the right hand side of $D_{2 k}$ can be obtained via

$$
\begin{aligned}
\int_{R^{2}} F_{1}^{k}(x) F_{2}^{k}(y) d F(x, y) & =\int_{R^{2}}\left\{\int_{-\infty}^{y} \int_{-\infty}^{x} d F_{1}^{k}(t) d F_{2}^{k}(s)\right\} d F(x, y) \\
& =\int_{R^{2}}\left\{\int_{s}^{\infty} \int_{t}^{\infty} d F(x, y)\right\} d F_{1}^{k}(t) d F_{2}^{k}(s) \\
& =\int_{R^{2}} \bar{F}(x, y) d F_{1}^{k}(y) d F_{2}^{k}(y) .
\end{aligned}
$$


Note that $\left(X_{1}, Y_{1}\right), \ldots,\left(X_{k+1}, Y_{k+1}\right)$ is a random sample of $(X, Y)$ with common joint distribution function $F(x, y)$. Now, it follows that under $H_{0}, D_{1 k}=D_{2 k}=1 /(k+1)^{2}$ and under $H_{1}$ we get,

$$
\begin{aligned}
D_{1 k} & =\int_{R^{2}} F^{k}(x, y) d F(x, y) \\
& \leq \int_{R^{2}} F_{1}^{k}(x) F_{2}^{k}(y) d F(x, y) \\
& =\int_{R^{2}} \bar{F}(x, y) d F_{1}^{k}(y) d F_{2}^{k}(y) \\
& <\int_{R^{2}} \bar{F}_{1}(x) \bar{F}_{2}(y) d F_{1}^{k}(y) d F_{2}^{k}(y)=\frac{1}{(k+1)^{2}} .
\end{aligned}
$$

Similarly under $H_{2}$, we get $D_{1 k}>1 /(k+1)^{2}$. So, if $H_{1}$ is true then, $D_{1 k}<D_{2 k}<1 /(k+1)^{2}$ and if $H_{2}$ is true then, $D_{1 k}>D_{2 k}>1 /(k+1)^{2}$ for all $k \geq 1$.

We use $U$-Statistics estimator of $D_{1 k}$ for testing $H_{0}$ against $H_{1}$ and $H_{2}$. Consider the following kernel according to Kochar and Gupta (1987):

$$
\begin{aligned}
& h_{k+1}\left[\left(X_{1}, Y_{1}\right), \ldots,\left(X_{k+1}, Y_{k+1}\right)\right] \\
& = \begin{cases}1, & \left(\max \left\{X_{1}, \ldots, X_{k+1}\right\}, \max \left\{Y_{1}, \ldots, Y_{k+1}\right\}\right) \text { belongs to the same pair of }(X, Y), \\
0, & \text { otherwise. }\end{cases}
\end{aligned}
$$

Then, we get

$$
\begin{aligned}
E h_{k+1}\left[\left(X_{1}, Y_{1}\right), \ldots,\left(X_{k+1}, Y_{k+1}\right)\right] & =(k+1) P\left[\max _{1 \leq i \leq k} X_{i} \leq X_{k+1}, \max _{1 \leq i \leq k} Y_{i} \leq Y_{k+1}\right] \\
& =(k+1) D_{1 k} .
\end{aligned}
$$

Therefore, the corresponding $U$-Statistics is

$$
U_{n}(k+1)=\frac{1}{C_{n}^{k+1}} \sum_{A} h_{k+1}\left[\left(X_{i_{1}}, Y_{i_{1}}\right), \ldots,\left(X_{i_{k+1}}, Y_{i_{k+1}}\right)\right],
$$

where $A$ is the set of all combinations of $(k+1)$ integers $\left(i_{1}, \ldots, i_{k+1}\right)$ that is chosen from $(1, \ldots, n)$ and $C_{n}^{i}=n ! /\{i !(n-i) !\}$.

Remark 1. The small values of $U_{n}(k+1)$ are significant for testing $H_{0}$ against $H_{1}$ (NQD) and the large values of $U_{n}(k+1)$ are significant for testing $H_{0}$ against $H_{2}$ (PQD).

The exact distribution of $U_{n}(k+1)$ can be simplified based on the following arguments,

$$
P_{n+1, k+1}(u)=\frac{1}{n+1}\left\{P_{n, k+1}\left(u-C_{n}^{k}\right)+\cdots+P_{n, k+1}\left(u-C_{k}^{k+1}\right)+P_{n, k+1}(u-1)+k P_{n, k+1}(u)\right\},
$$

where $P_{n, k+1}(u)=P_{H_{0}}\left[U_{n}(k+1)=u / C_{n}^{k+1}\right]$ and $P_{k, k}(0)=(k-1) / k, P_{k, k}(1)=1 / k$ for $n>k,($ Kochar and Gupta, 1987).

Now, we concentrate on asymptotic distribution of $U_{n}(k+1)$ that is achieved based on asymptotic theory of $U$-statistics via the the following theorem. 
Theorem 1. The asymptotic distribution of $\sqrt{n}\left(U_{n}(k+1)-E U_{n}(k+1)\right)$ is normal with mean zero and $\sigma_{k+1}^{2}=(k+1)^{2} \gamma_{1}$ as $n \longrightarrow \infty$ where,

$$
\gamma_{1}=E \psi^{2}\left(X_{1}, Y_{1}\right)-E^{2} U_{n}(k+1)
$$

and

$$
\psi\left(x_{1}, y_{1}\right)=E h_{k+1}\left[\left(x_{1}, y_{1}\right),\left(X_{2}, Y_{2}\right), \ldots,\left(X_{k+1}, Y_{k+1}\right)\right] .
$$

Proof: See, Serfling (1980).

Remark 2. Let $\left(X_{1}, Y_{1}\right), \ldots,\left(X_{k+1}, Y_{k+1}\right)$ be a random sample of $(X, Y)$ with common joint distribution function $F(x, y)$, we get

$$
\begin{aligned}
\psi\left(x_{1}, y_{1}\right) & =E h_{k+1}\left[\left(x_{1}, y_{1}\right),\left(X_{2}, Y_{2}\right), \ldots,\left(X_{k+1}, Y_{k+1}\right)\right] \\
& =F^{k}\left(x_{1}, y_{1}\right)+k \cdot P\left[\max \left\{x_{1}, \ldots, X_{k}\right\} \leq X_{k+1}, \max \left\{y_{1}, \ldots, Y_{k}\right\} \leq Y_{k+1}\right] \\
& =F^{k}\left(x_{1}, y_{1}\right)+k \cdot P\left[\max _{2 \leq i \leq k} X_{i} \leq X_{k+1}, \max _{2 \leq i \leq k} Y_{i} \leq Y_{k+1},\left(X_{k+1} \geq x_{1}, Y_{k+1} \geq y_{1}\right)\right] \\
& =F^{k}\left(x_{1}, y_{1}\right)+k \int_{y_{1}}^{\infty} \int_{x_{1}}^{\infty} F^{k-1}(x, y) d F(x, y) .
\end{aligned}
$$

Thus, under $H_{0}$, we obtain

$$
\begin{aligned}
\psi\left(x_{1}, y_{1}\right) & =F_{1}^{k}\left(x_{1}\right) F_{2}^{k}\left(y_{1}\right)+k \int_{y_{1}}^{\infty} \int_{x_{1}}^{\infty} F_{1}^{k-1}(x) F_{2}^{k-1}(y) d F_{1}(x) d F_{2}(y) \\
& =F_{1}^{k}\left(x_{1}\right) F_{2}^{k}\left(y_{1}\right)+\frac{1}{k}\left[1-F_{1}^{k}\left(x_{1}\right)\right]\left[1-F_{2}^{k}\left(y_{1}\right)\right] .
\end{aligned}
$$

Since $F_{1}\left(X_{1}\right) \sim U(0,1)$ and $F_{2}\left(Y_{1}\right) \sim U(0,1)$, we have

$$
\begin{aligned}
E_{H_{0}} \psi\left(X_{1}, Y_{1}\right) & =E F_{1}^{k}\left(X_{1}\right) E F_{2}^{k}\left(Y_{1}\right)+\frac{1}{k} E\left[1-F_{1}^{k}\left(X_{1}\right)\right] E\left[1-F_{2}^{k}\left(Y_{1}\right)\right] \\
& =\frac{1}{k+1}=E_{H_{0}} U_{n}(k+1)
\end{aligned}
$$

and

$$
\begin{aligned}
E_{H_{0}} \psi^{2}\left(X_{1}, Y_{1}\right) & =E\left\{F_{1}^{k}\left(X_{1}\right) F_{2}^{k}\left(Y_{1}\right)+\frac{1}{k}\left[1-F_{1}^{k}\left(X_{1}\right)\right]\left[1-F_{2}^{k}\left(Y_{1}\right)\right]\right\}^{2} \\
& =\frac{5 k^{2}+4 k+1}{(2 k+1)^{2}(k+1)^{2}} .
\end{aligned}
$$

So,

$$
\gamma_{1}=E_{H_{0}} \psi^{2}\left(X_{1}, Y_{1}\right)-E_{H_{0}}^{2} U_{n}(k+1)=\frac{k^{2}}{(2 k+1)^{2}(k+1)^{2}}
$$

and under $H_{0}$, for large values of $n$, Lemma 5.2.1.A in Serfling (1980) implies that

$$
n \cdot \operatorname{Var}\left\{U_{n}(k+1)\right\}=\frac{k^{2}}{(2 k+1)^{2}}, \quad \text { as } n \rightarrow \infty .
$$


Remark 3. The estimator of $U_{n}(2)$ is Kendall's tau statistics, because

$$
U_{n}(2)=\frac{1}{C_{n}^{2}} \sum_{A} h_{2}\left[\left(X_{i}, Y_{i}\right),\left(X_{j}, Y_{j}\right)\right],
$$

where $A=\left\{(i, j):\left(X_{j}-X_{i}\right)\left(Y_{j}-Y_{i}\right)>0\right\}$.

\subsection{Bivariate FGM family}

Morgenstern (1956), Farlie (1960) and Gumbel (1958) have discussed family of the bivariate distributions of the form

$$
F(x, y)=F_{1}(x) F_{2}(y)\left[1+\theta \bar{F}_{1}(x) \bar{F}_{2}(y)\right], \quad-1 \leq \theta \leq 1,
$$

where $F(x, y)$ is the joint distribution function of $(X, Y)$ and $F_{1}$ and $F_{2}$ are marginal distribution functions of $X$ and $Y$, respectively. If the density function $f(x, y)$ exists, then

$$
f(x, y)=f_{1}(x) f_{2}(y)\left[1+\theta\left(1-2 F_{1}(x)\right)\left(1-2 F_{2}(y)\right)\right]
$$

and Sklar's theorem implies that in FGM family for $-1 \leq \theta \leq 1$

$$
C(u, v)=u v(1+\theta(1-u)(1-v))
$$

and

$$
c(u, v)=1+\theta(1-2 u)(1-2 v),
$$

where $C(u, v)$ and $c(u, v)$ are copula distribution function and copula density function, respectively. For more details and properties, see Mari and Kotz (2001). The following example shows an empirical application of FGM family.

Example 1. Consider a two-component system such as desktop computer with both a CPU(central processing unit) and a co-processor. Let $X$ and $Y$ denote the lifetimes of the components 1 and 2 , respectively. As is often the case in dealing with lifetimes, the components are related together. So the amount of using the first component has negative (positive) effects on the lifetimes of the second one. If $X$ and $Y$ having every common distribution, the dependence structure of them can be determined with FGM family. Also according to the properties of copula function, the dependence structure of $X$ and $Y$ is not dependent to the distribution of them.

\subsection{Bivariate generalized FGM family}

The bivariate FGM distribution has been modified by Bairamov and Kotz (2002) in order to increase the dependence between the variables. The modified bivariate FGM distribution is known as generalized FGM distribution. If $(X, Y)$ be a pair of absolutely continuous random variables with the marginals $F_{1}(x)$ and $F_{2}(y)$, the generalized FGM distribution function of $(X, Y)$ is

$$
F(x, y)=F_{1}(x) F_{2}(y)\left[1+\theta\left(1-F_{1}^{p}(x)\right)^{q}\left(1-F_{2}^{p}(y)\right)^{q}\right], \quad p \geq 1, q \geq 1,
$$

where $\theta$ is the dependence parameter. Note that assuming $p=1$ and $q=1$, lead to FGM distribution.

Let $\left(X_{i}, Y_{i}\right), i=1,2, \ldots, n$ be a random sample from the generalized FGM distribution. Güven and Kotz (2008) proposed an asymptotic test for testing independence $\left(H_{0}: \theta=0\right)$ against quadrant 
Table 1: Estimated power for marginals $U(0,1)$ in FGM family for NQD cases

\begin{tabular}{ccccccc}
\hline \hline \multirow{2}{*}{$n$} & $U$ & $5 \%$ Critical & & \multicolumn{3}{c}{$\theta$} \\
\cline { 4 - 7 } & Statistics & Point & -0.75 & -0.5 & -0.25 & 0.00 \\
\hline \multirow{2}{*}{6} & $U_{n}(2)$ & 0.20000 & 0.06500 & 0.05087 & 0.03670 & 0.02833 \\
& $U_{n}(3)$ & 0.05000 & $\mathbf{0 . 0 9 4 0 0}$ & $\mathbf{0 . 0 7 4 2 3}$ & $\mathbf{0 . 0 5 6 3 5}$ & 0.04483 \\
& $U_{n}(4)$ & 0.00000 & 0.00000 & 0.00000 & 0.00000 & 0.00000 \\
\hline \multirow{2}{*}{8} & $U_{n}(2)$ & 0.25000 & 0.08665 & 0.06377 & 0.04612 & 0.03055 \\
& $U_{n}(3)$ & 0.07143 & $\mathbf{0 . 1 0 4 3 8}$ & $\mathbf{0 . 0 7 7 7 3}$ & $\mathbf{0 . 0 5 8 4 0}$ & 0.04057 \\
& $U_{n}(4)$ & 0.01429 & 0.08948 & 0.066840 & 0.05185 & 0.03648 \\
\hline \multirow{2}{*}{10} & $U_{n}(2)$ & 0.28889 & 0.12088 & 0.08252 & 0.05563 & 0.03533 \\
& $U_{n}(3)$ & 0.10833 & $\mathbf{0 . 1 4 9 6 3}$ & $\mathbf{0 . 1 0 5 8 7}$ & $\mathbf{0 . 0 7 4 9 3}$ & 0.04947 \\
& $U_{n}(4)$ & 0.02857 & 0.13702 & 0.09875 & 0.07067 & 0.04747 \\
\hline \multirow{2}{*}{12} & $U_{n}(2)$ & 0.31818 & 0.16100 & 0.10712 & 0.06935 & 0.04182 \\
& $U_{n}(3)$ & 0.12727 & $\mathbf{0 . 1 6 4 8 5}$ & $\mathbf{0 . 1 1 2 0 8}$ & 0.07513 & 0.04630 \\
& $U_{n}(4)$ & 0.04646 & 0.15923 & 0.10985 & $\mathbf{0 . 0 7 5 4 7}$ & 0.04933 \\
\hline \multirow{2}{*}{16} & $U_{n}(2)$ & 0.35000 & $\mathbf{0 . 2 1 3 8 5}$ & 0.13947 & 0.08502 & 0.04505 \\
& $U_{n}(3)$ & 0.16071 & 0.21093 & $\mathbf{0 . 1 4 0 6 5}$ & $\mathbf{0 . 0 8 8 7 0}$ & 0.0499 \\
\hline \multirow{2}{*}{20} & $U_{n}(4)$ & 0.07308 & 0.18977 & 0.12935 & 0.08358 & 0.04977 \\
\hline \hline & $U_{n}(2)$ & 0.36842 & $\mathbf{0 . 2 6 0 1 2}$ & $\mathbf{0 . 1 6 0 8 0}$ & $\mathbf{0 . 0 9 1 3 3}$ & 0.04907 \\
& $U_{n}(3)$ & 0.17982 & 0.24650 & 0.15653 & 0.08917 & 0.04937 \\
\hline
\end{tabular}

dependence $\left(H_{1}: \theta \geq \eta\right.$ or $\left.H_{2}: \theta \leq \eta\right)$ for this family, where $\eta \in(0,1)$. Moreover, they proved that the generalized FGM family having monotone likelihood ratio in

$$
\omega(x, y)=\left(1-x^{p}\right)^{q-1}\left(1-y^{p}\right)^{q-1}\left(1-(1+p q) x^{p}\right)\left(1-(1+p q) y^{p}\right)
$$

and so the test statistics are

$$
\varphi(T)= \begin{cases}1, & \text { if } T \geq c, \\ 0, & \text { if } T<c,\end{cases}
$$

where $T=\prod_{i=1}^{n}\left(1+\eta \omega\left(X_{i}, Y_{i}\right)\right)$. Also, they showed that under $H_{0}$

$$
\sqrt{n}\left(T^{\frac{1}{n}}-\mu(\theta)\right) \rightarrow N\left(0, \sigma^{2}(\theta)\right), \quad \text { as } n \rightarrow \infty,
$$

where $\mu(\theta)=\exp [E \ln (1+\eta \omega(X, Y))]$ and $\sigma^{2}(\theta)=\mu^{2}(\theta) \operatorname{Var}[\ln (1+\eta \omega(X, Y))]$. They obtained an approximation to $\beta_{n}(\theta)=E \varphi(T)$ for a finite sample given by $\beta_{n}(\theta)=1-\Phi\left(\sqrt{n} / \sigma(\theta)\left[c_{\alpha}-\mu(\theta)\right]\right)$, where $\theta \in[\eta, 1], c_{\alpha}=\sigma(0) / \sqrt{n} \Phi^{-1}(1-\alpha)+\mu(0)$ and $\Phi(\cdot)$ is the standard normal distribution function.

\section{Main Results}

In this section, we consider the problem of test independence hypothesis against NQD (PQD) in absolutely continuous bivariate FGM family via comparing the power of the tests based on simulation results. We compute the $5 \%$ critical point of the tests $U_{n}(2), U_{n}(3)$ and $U_{n}(4)$ and their empirical powers based on 60000 repetitions in FGM family using R software version 2.10.1 (2009-12-14). The critical points and empirical powers for NQD and PQD cases are summarized in Tables 1 and 2, respectively. Also, the power of $U_{n}(k+1), k=1,2,3$ and $T$ based on their asymptotic distribution for sample of sizes $n=10,14,16,20,50$ with various values of $\eta \in(0,1)$ and $\theta \in[\eta, 1]$ at $5 \%$ level of significance using R software version 2.10 .1 (2009-12-14) is obtained. Figures 1, 2, 3, 4 and 5 show the powers comparison for $n=10,14,16,20,50$ and specific values of $\eta$ with respect to $\theta$. 
Table 2: Empirical power for marginals $U(0,1)$ in FGM family for PQD cases

\begin{tabular}{ccccccc}
\hline \hline \multirow{2}{*}{$n$} & $U$ & $5 \%$ Critical & & \multicolumn{3}{c}{$\theta$} \\
\cline { 3 - 7 } & Statistics & Point & 0.00 & 0.25 & 0.5 & 0.75 \\
\hline \multirow{2}{*}{6} & $U_{n}(2)$ & 0.80000 & 0.02830 & 0.03868 & 0.04925 & 0.06638 \\
& $U_{n}(3)$ & 0.75000 & 0.04432 & $\mathbf{0 . 0 5 7 8 5}$ & $\mathbf{0 . 0 7 2 8 3}$ & $\mathbf{0 . 0 9 3 0 2}$ \\
& $U_{n}(4)$ & 0.73333 & 0.04165 & 0.05382 & 0.06737 & 0.08587 \\
\hline \multirow{2}{*}{8} & $U_{n}(2)$ & 0.75000 & 0.03153 & 0.04582 & 0.06357 & 0.08867 \\
& $U_{n}(3)$ & 0.66071 & 0.04345 & 0.06000 & 0.08215 & $\mathbf{0 . 1 0 9 1 2}$ \\
& $U_{n}(4)$ & 0.64286 & 0.04715 & $\mathbf{0 . 0 6 2 0 3}$ & $\mathbf{0 . 0 8 2 6 7}$ & 0.10352 \\
\hline \multirow{2}{*}{10} & $U_{n}(2)$ & 0.71111 & 0.03555 & 0.05717 & 0.08242 & 0.12072 \\
& $U_{n}(3)$ & 0.60833 & 0.04528 & 0.06720 & $\mathbf{0 . 0 9 4 4 8}$ & $\mathbf{0 . 1 3 2 3 7}$ \\
& $U_{n}(4)$ & 0.57619 & 0.04873 & $\mathbf{0 . 0 6 8 0 0}$ & 0.09183 & 0.12220 \\
\hline \multirow{2}{*}{12} & $U_{n}(2)$ & 0.68182 & 0.04343 & 0.07073 & 0.10732 & $\mathbf{0 . 1 5 8 4 7}$ \\
& $U_{n}(3)$ & 0.57273 & 0.04870 & $\mathbf{0 . 0 7 4 0 0}$ & $\mathbf{0 . 1 0 7 4 7}$ & 0.15313 \\
& $U_{n}(4)$ & 0.53333 & 0.04888 & 0.07005 & 0.09780 & 0.13435 \\
\hline \multirow{2}{*}{16} & $U_{n}(2)$ & 0.65000 & 0.04785 & 0.08480 & $\mathbf{0 . 1 3 7 5 3}$ & $\mathbf{0 . 2 1 6 6 3}$ \\
& $U_{n}(3)$ & 0.52679 & 0.04985 & $\mathbf{0 . 0 8 5 5 7}$ & 0.13370 & 0.20487 \\
\hline \multirow{2}{*}{20} & $U_{n}(4)$ & 0.47527 & 0.04982 & 0.07963 & 0.11767 & 0.17073 \\
\hline \hline & $U_{n}(2)$ & 0.63158 & 0.04860 & $\mathbf{0 . 0 9 3 4 5}$ & $\mathbf{0 . 1 6 4 7 3}$ & $\mathbf{0 . 2 6 4 1 2}$ \\
& $U_{n}(3)$ & 0.50088 & 0.04958 & 0.09287 & 0.15742 & 0.24528 \\
\hline
\end{tabular}

\subsection{Conclusions}

The following results are observed:

- We applied the class of distribution-free tests (Kochar and Gupta, 1987) for testing independence against quadrant dependence, so selecting various marginals in the FGM family give the same results. We have done this for various of margins and observed our results are the same. Therefore, we use FGM family for margins $U(0,1)$ and $U(0,1)$. Tables 1 and 2 show the empirical powers of $U_{n}(i), i=2,3,4$ for marginals $U(0,1)$ and $U(0,1)$ in NQD and PQD cases, respectively.

Comparing the powers of the tests $U_{n}(i), i=2,3,4$ based on simulation study in PQD case $(0<\theta \leq 1)$ and NQD $(-1 \leq \theta<0)$ indicates that:

- For PQD $(\theta=0.25,0.5,0.75)$ when $n=6$, test $U_{n}(3)$ is a good competitor of tests $U_{n}(2)$ and $U_{n}(4)$. When $n=8, U_{n}(4)$ is a good competitor of the other tests except for $\theta=0.75$ that $U_{n}(3)$ is a good competitor test, note that the power of $U_{n}(3)$ is very close to the corresponding value due to $U_{n}(4) . n=10,12$ lead to $U_{n}(3)$ is a good competitor of tests $U_{n}(2)$ and $U_{n}(4)$ except for $n=12$ in $\theta=0.75, U_{n}(2)$ and for $n=10$ in $\theta=0.25, U_{n}(4)$ are better than other tests. Noting that in these cases the power of the best test statistics is very close to those of their competitors. When $n=16,20, U_{n}(2)$ test is a good competitor of $U_{n}(3)$ and $U_{n}(4)$, except when $n=16$ for $\theta=0.25$ that $U_{n}(3)$ is a good competitor but also its power test values are very close to the corresponding values due to $U_{n}(2)$.

- In NQD cases, for $\theta=-0.75,-0.5,-0.25$, when $n=6,8,10,12, U_{n}(3)$ test is a good competitor of $U_{n}(2)$ and $U_{n}(4)$ tests, except when $n=12$, for $\theta=-0.25$ that $U_{n}(4)$ is a good competitor test, note that the power test value of $U_{n}(4)$ is very close to the corresponding value due to $U_{n}(3)$. When $n=16,20, U_{n}(2)$ is a good competitor of $U_{n}(3)$ and $U_{n}(4)$ tests, except when $n=16$ the test $U_{n}(3)$ is better than $U_{n}(2)$ and $U_{n}(4)$ for $\theta=-0.5,-0.25$.

- Comparison of our results with results of Güven and Kotz (2008) based on asymptotic distribution of $T$ in FGM family for $p=q=1$, leads to the following results: 

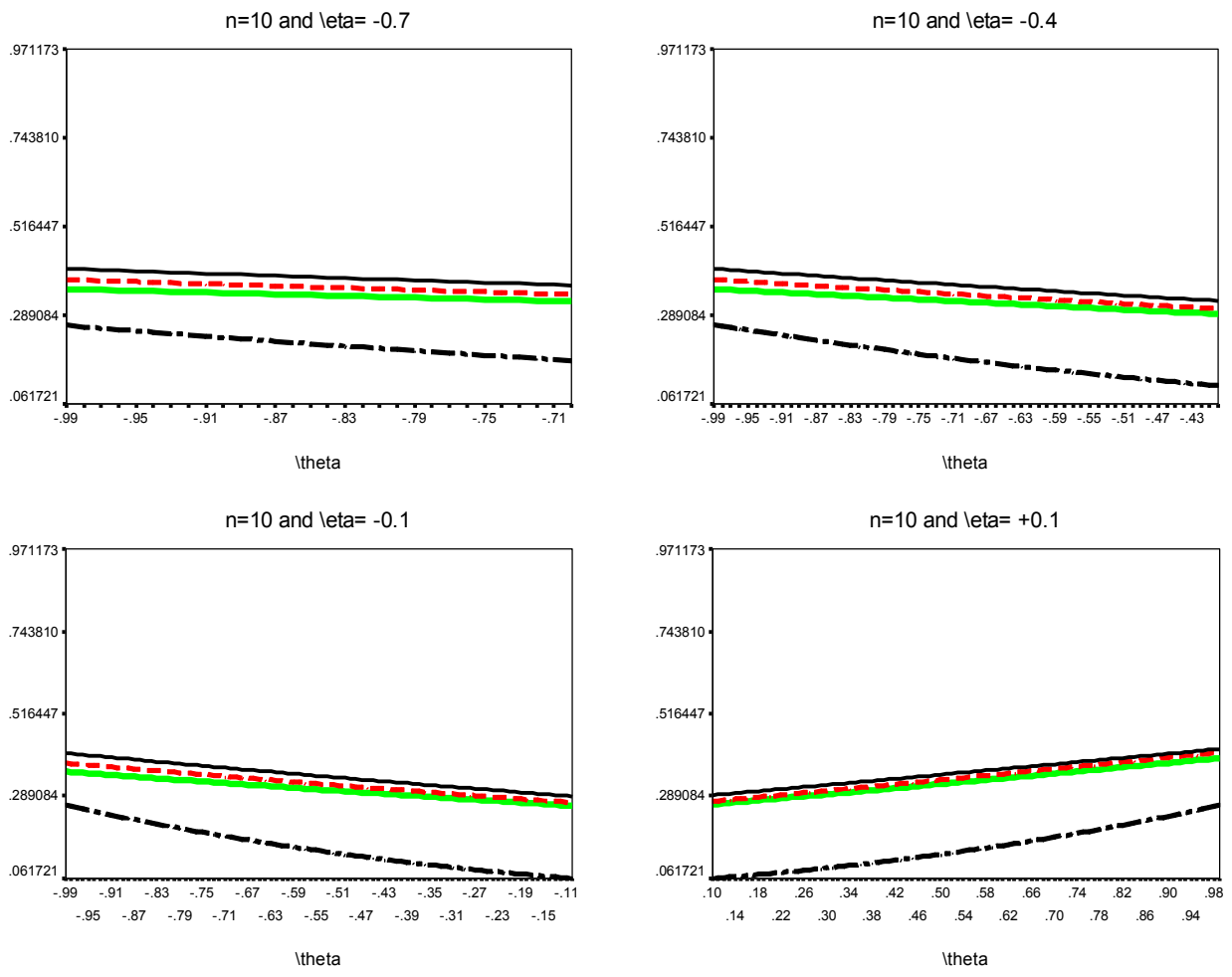

$\mathrm{n}=10$ and $l$ eta $=+0.4$
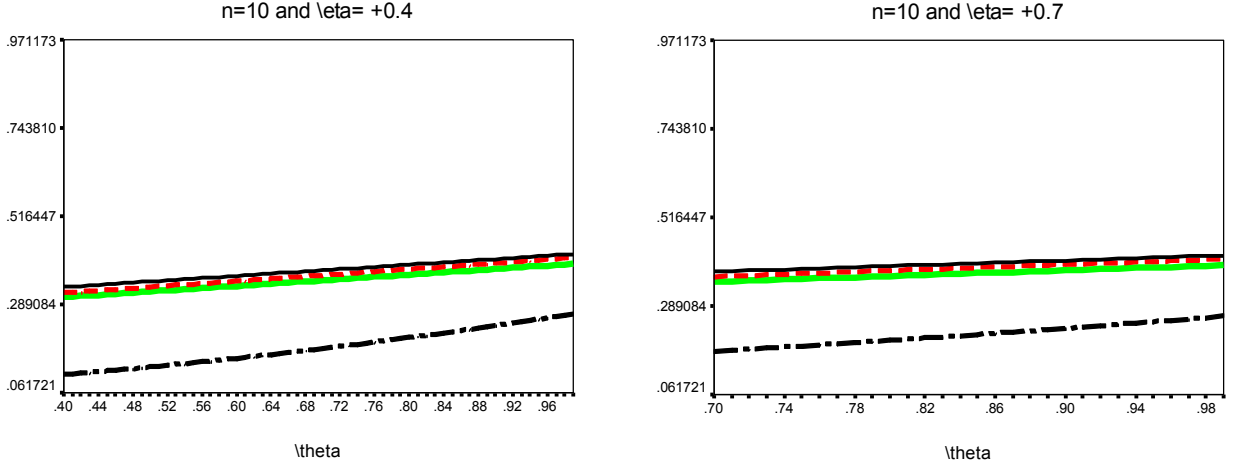

Figure 1. The power of $U_{n}(2), U_{n}(3), U_{n}(4)$ and $T$ with respect to $\theta$ for $n=10$ and $\eta=-0.7,-0.4,-0.1,+0.1,+0.4,+0.7$.

In all graphs _ _, - , _ and _ . _ show the power of $U_{n}(2), U_{n}(3), U_{n}(4)$ and $T$, respectively.

a) Figures 1, 2, 3 and $4(n=10,14,16,20)$ show that the power of the tests based on $U_{n}(i), i=$ 2,3,4 are greater than $T$ in NQD and PQD cases. Figure $5(n=50)$ shows that the power of $T$ is better than others, when $|\eta|$ has a large value. Also, we observe that when $|\eta|$ is not large and $\theta$ is decreased, $U_{n}(i), i=2,3,4$ are better than $T$.

b) The tests $U_{n}(i), i=2,3,4$ for small samples less than 50 are better than the asymptotic test 

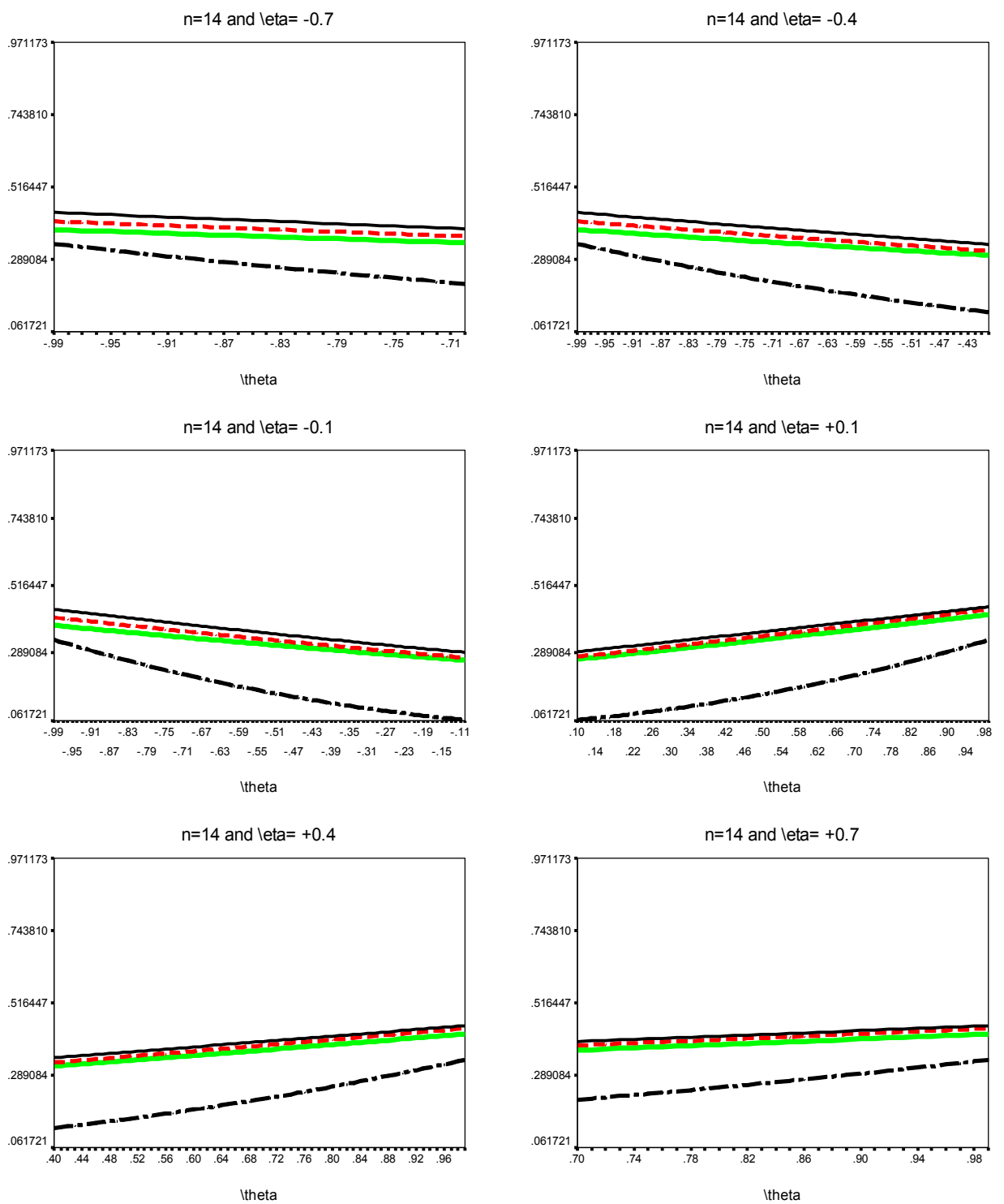

Figure 2. The power of $U_{n}(2), U_{n}(3), U_{n}(4)$ and $T$ with respect to $\theta$ for $n=14$ and $\eta=-0.7,-0.4,-0.1,+0.1,+0.4,+0.7$.

In all graphs _ $, \ldots, \ldots$ and $\ldots$ - show the power of $U_{n}(2), U_{n}(3), U_{n}(4)$ and $T$, respectively.

of Güven and Kotz (2008). Moreover for large samples ( $n \geq 50)$, our results show that the tests $U_{n}(i), i=2,3,4$ are better than the test of Güven and Kotz (2008), when $|\theta|$ is decreased and $|\eta|$ is not large.

- Finally, Tables 1 and 2 and Figures 1, 2, 3, 4 and 5 show that: 

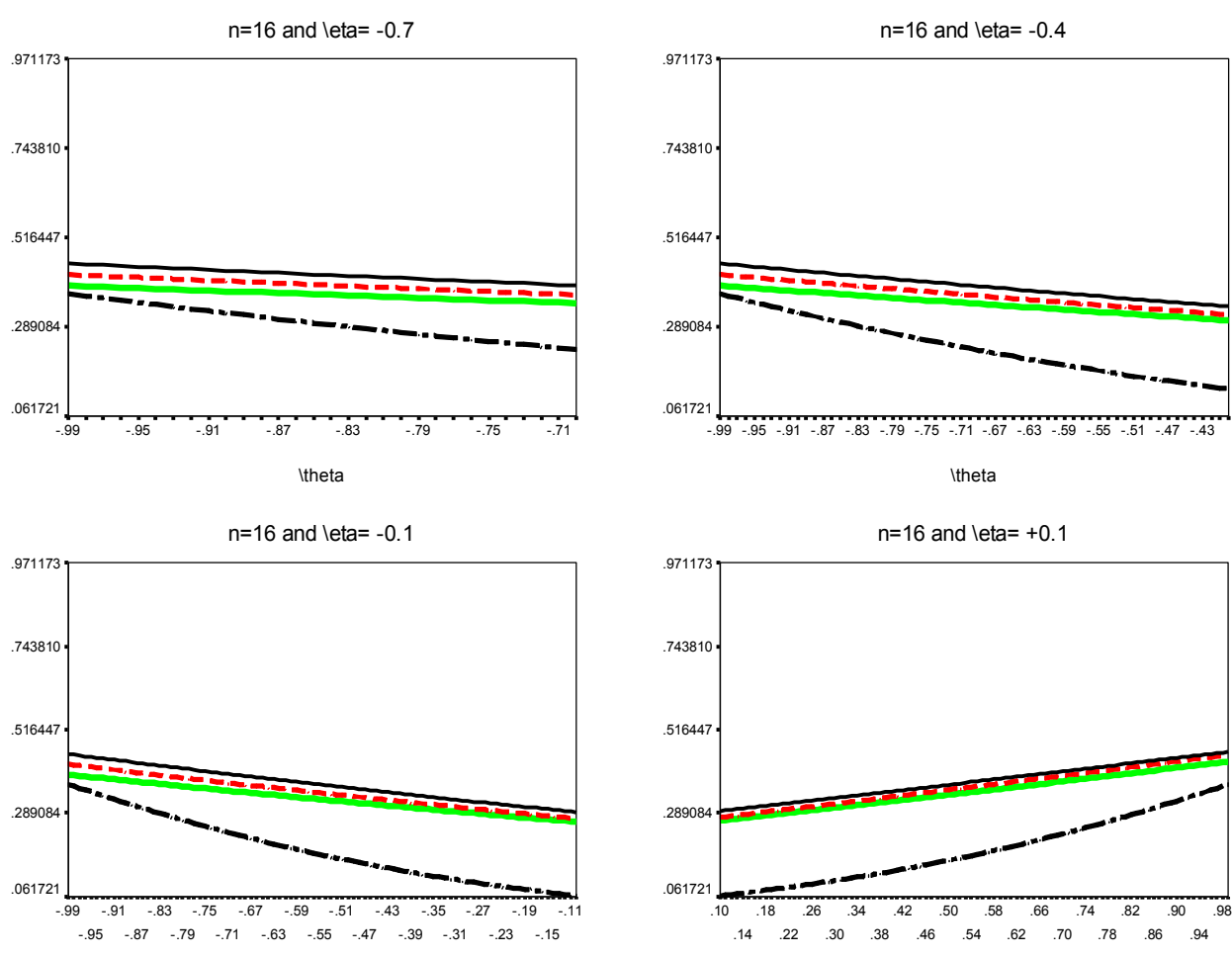

thet
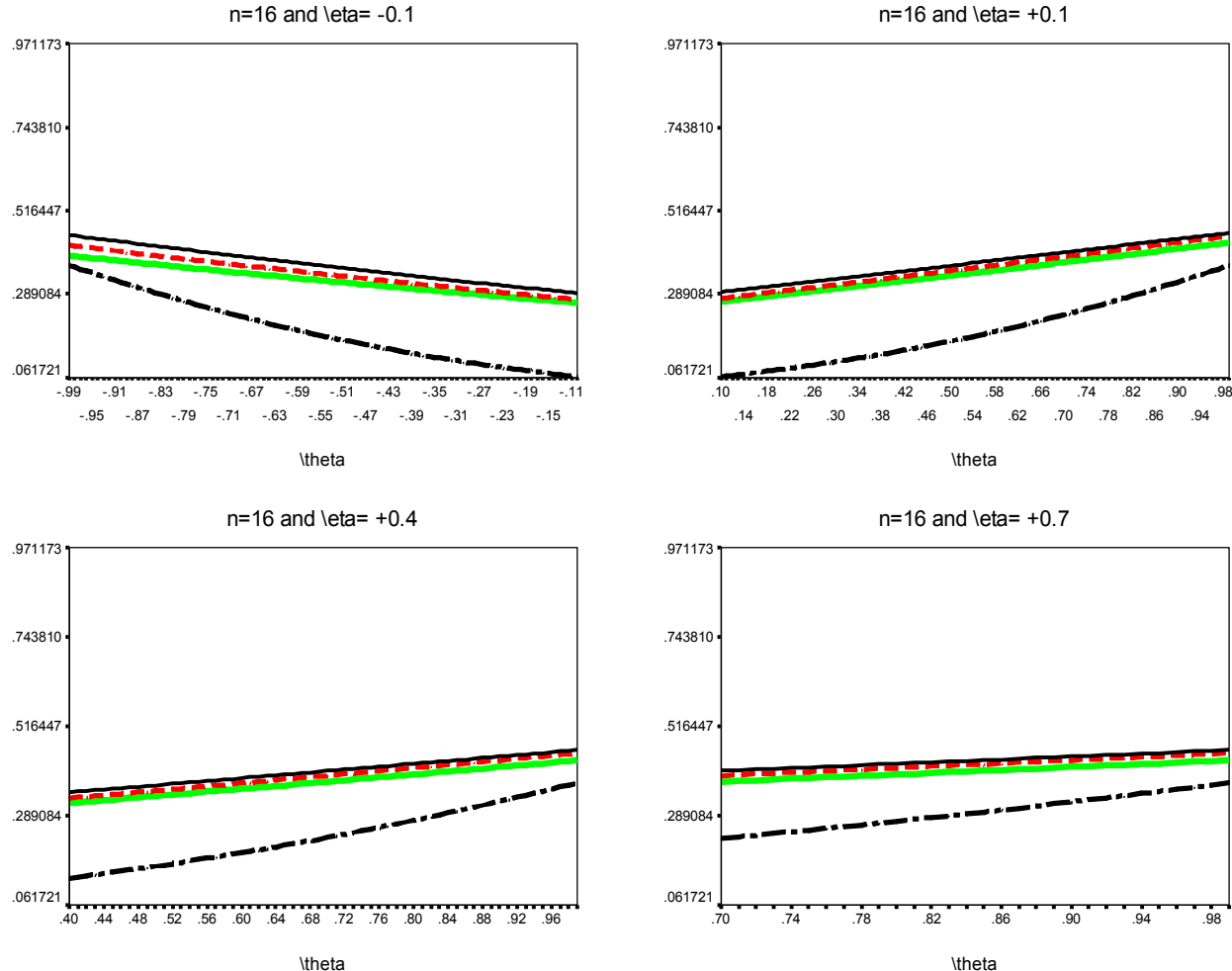

Figure 3. The power of $U_{n}(2), U_{n}(3), U_{n}(4)$ and $T$ with respect to $\theta$ for $n=16$ and $\eta=-0.7,-0.4,-0.1,+0.1,+0.4,+0.7$.

In all graphs _ $,-\ldots, \ldots$ and $\ldots .-$ show the power of $U_{n}(2), U_{n}(3), U_{n}(4)$ and $T$, respectively.

For small samples, $U_{n}(2)$ is not a good statistics for testing independence in both NQD and PQD cases.

For large samples, $U_{n}(3)$ or $U_{n}(4)$ is not a good statistics for testing independence in both NQD and PQD cases. 

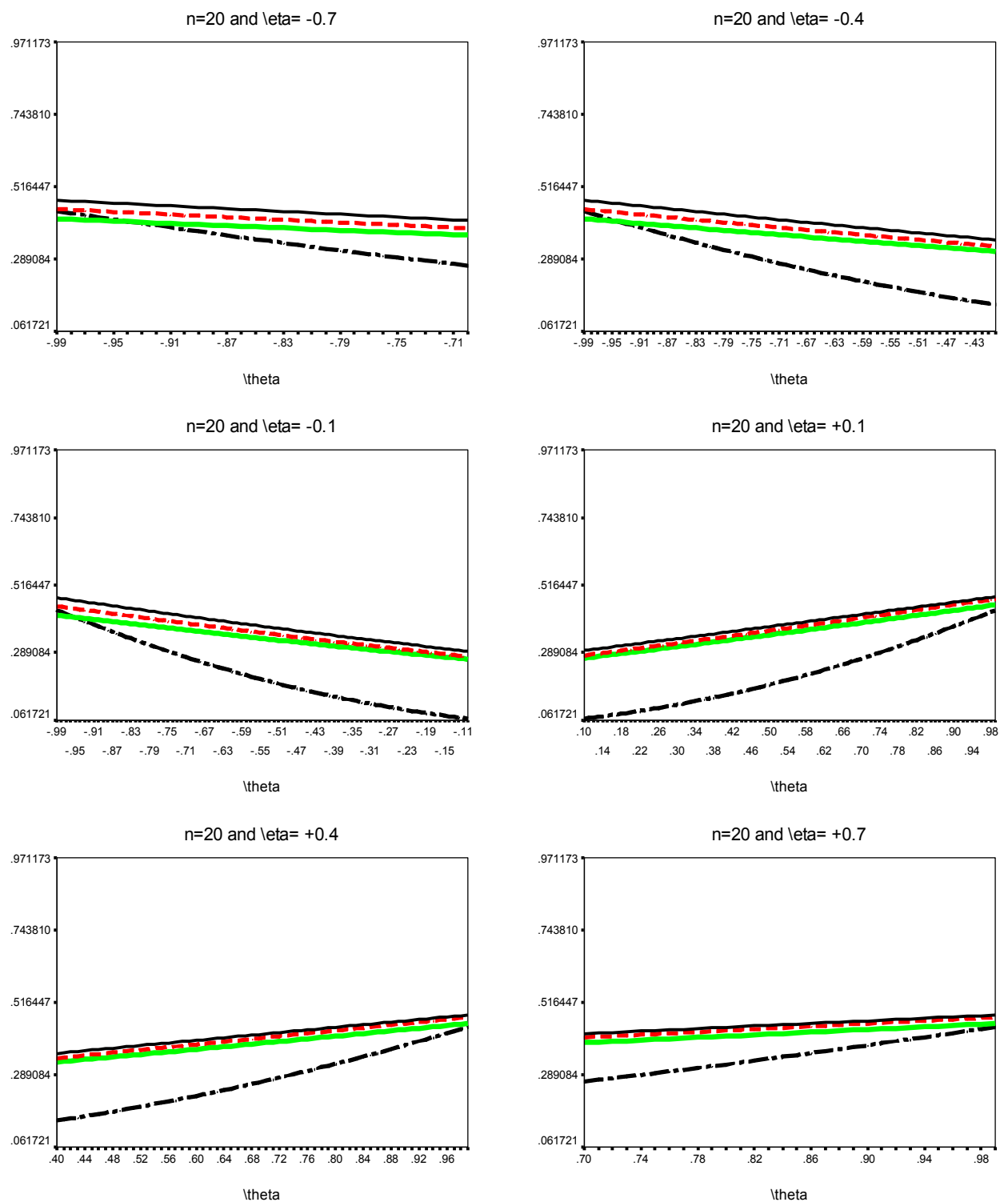

Figure 4. The power of $U_{n}(2), U_{n}(3), U_{n}(4)$ and $T$ with respect to $\theta$ for $n=20$ and $\eta=-0.7,-0.4,-0.1,+0.1,+0.4,+0.7$.

In all graphs _ , - - , — and - . - show the power of $U_{n}(2), U_{n}(3), U_{n}(4)$ and $T$, respectively.

Since for computing $U_{n}(4)$ or $U_{n}(3)$ we used the fourth or triangle combinations of the observation respectively, the above results are satisfied. So for large samples, the power of $U_{n}(2)$ and its competitors are the same for evaluating the dependence between two random variables.

- Applying these methods for other bivariate distributions is to be done in a future study. Also, the 

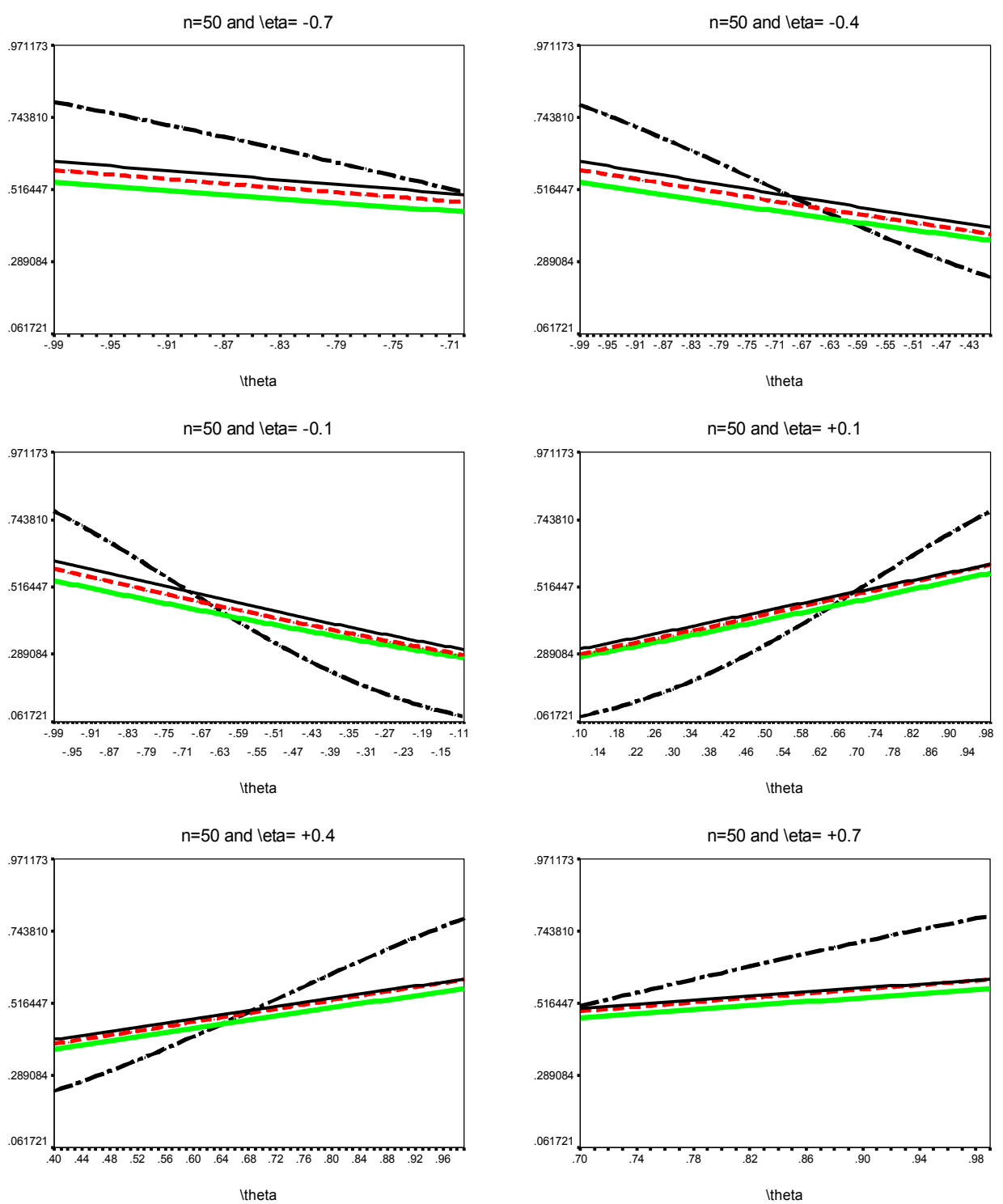

Figure 5. The power of $U_{n}(2), U_{n}(3), U_{n}(4)$ and $T$ with respect to $\theta$ for $n=50$ and $\eta=-0.7,-0.4,-0.1,+0.1,+0.4,+0.7$.

In all graphs _,,$-- \ldots$ and $\ldots$ - show the power of $U_{n}(2), U_{n}(3), U_{n}(4)$ and $T$, respectively.

tests $U_{n}(i)$ for $k>3$ is another open problem that can be solve in the future.

\section{Acknowledgement}

The authors would like to thank the referees for their careful reading of the manuscript and for many valuable suggestions which improved the presentation of the paper. 


\section{References}

Bairamov, I. and Kotz, S. (2002). Dependence structure and symmetry of Huang-Kotz FGM distributions and their extensions, Metrika, 56, 55-72.

Block, H. W. and Basu, A. P. (1974). A continuous bivariate exponential extension, Journal of the American Statistical Association, 69, 1031-1037.

Farlie, D. J. G. (1960). The performance of some correlation coefficients for a general bivariate distribution function, Biometrika, 47, 307-323.

Gibbons, J. D. (1971). Nonparametric Statistical Inference, MaGraw-Hill.

Gumbel, E. J. (1958). Statistics of Extremes, Columbia University Press, New York.

Güven, B. and Kotz, S. (2008). Test of independence for generalized Farlie-Gumbel-Morgenstern distributions, Journal of Computational and Applied Mathemathics, 212, 102-111.

Hanagal, D. D. and Kale, B. K. (1991). Large sample tests of independence for absolutely continuous bivariate exponential distribution, Communications in Statistics - Theory and Methods, 20, 13011313.

Kochar, S. G. and Gupta, R. P. (1987). Competitors of Kendall-tau test for testing independence against PQD, Biometrika, 74, 664-669.

Kochar, S. G. and Gupta, R. P. (1990). Distribution-free tests based on sub-sample extrema for testing against positive dependence, Australian Journal of Statistics, 32, 45-51.

Koroljuk, V. S. and Borovskich, Y. V. (1994). Theory of U-statistic, Kluwer Academic Publishers.

Lehmann, E. L. (1966). Some concepts of dependence, The Annals of Mathematical Statistics, 37, 1137-1153.

Mari, D. D. and Kotz, S. (2001). Correlation and Dependence, Imperical College Press.

Modarres, R. (2007). A test of independence based on the likelihood of Cut-Points, Communicationa in Statistics-Simulation and Computation, 36, 817-825.

Morgenstern, D. (1956). Einfache Beispiele Zweidimensionaler Verteilungen, Mitteilungsblatt für Mathematische Statistik, 8, 234-235.

Serfling, R. J. (1980). Approximations Theorems of Mathematical Statistics, John Wiley \& Sons.

Shetty, I. D. and Pandit, P. V. (2003). Distribution-free tests for independence against positive quadrant dependence: A generalization, Statistical Methods and Application, 12, 5-17. 